\title{
MG2016+112: A DOUBLE GRAVITATIONAL LENS MODEL
}

\author{
S. NAIR AND M. A. GARRETT \\ University of Manchester, NRAL, Jodrell Bank, U.K.
}

MG2016+112, discovered by Lawrence et al. (1984) is one of the beststudied among multiply-imaged systems, but is still only partially understood. Ostensibly a three-image system consisting of images $\mathrm{A}, \mathrm{B}$ and $\mathrm{C}$ of a quasar at $z=3.273$, the observed lensing galaxy $\mathrm{D}$ (a giant elliptical at $z=1.01)$ at the centroid of the image system seems inadequate to provide the minimum mass of $\sim 2.5 \times 10^{12} \mathrm{M}_{\odot}$ within $10 \mathrm{kpc}$ of its center (in projection along the 1.o.s.) required to produce the observed 3." 9 image-splitting. C itself appears to consist of two components, radio emission that may be associated with the faint optical image counterpart of $\mathrm{A}$ and $\mathrm{B}$ (called $\mathrm{C}_{2}$, see Garrett et al. 1994) and flat-spectrum $\mathrm{C}_{1}$, which dominates radio observations of the system and apparently consists of at least three linearly stretched subcomponents, $\mathrm{C}_{11}$ to $\mathrm{C}_{13}$ (see Garrett et al. in these proceedings).

The observationally suggested second lens in region C (e.g. Lawrence et al. 1993) is strongly supported by lens modeling, because of the presence of the faint image $\mathrm{C}_{2}$. The two elliptical lenses produce a five-image configuration with two core-captured images demagnified to levels of undetectability; see Fig.1. Each lens consists of two non-singular oblate spheroidal mass distributions, one compact (the 'galaxy') and the other extended ('dark matter', DM). The DM associated with lens plane D has a scale length of about $25 \mathrm{kpc}$, and appears to have a high eccentricity (axial ratio about 0.6 in a typical model). Hattori et al. (these proceedings) suggest that there could be a cluster here. The redshift of lens plane $\mathrm{C}$ is assumed to be greater than that of $\mathrm{D}$, for definiteness (this is not constrained by the configuration). Masses (in $\mathrm{M}_{\odot}$ ) of the lenses in the present model are: lens plane D: galaxy $-2.7 \times 10^{11}, \mathrm{DM}-2.3 \times 10^{12}$; lens plane $\mathrm{C}$ : galaxy $-6.5 \times 10^{9}$ (high eccentricity), $\mathrm{DM}-7.0 \times 10^{12}$ (scale length of $\sim 65 \mathrm{kpc}$; spherical). Lens plane $\mathrm{C}$ is at $z_{C}=1.2$, and lens plane $\mathrm{D}$ is at $z_{D}=1.01$. The modeling code used is a version of Narasimha, Subramanian and Chitre $(1982,1984)$. 

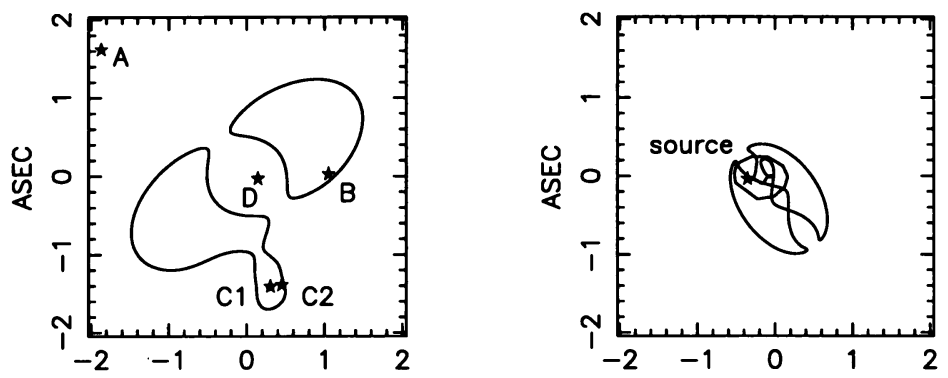

Figure 1. (a) Image and (b) Source Planes for the Two-Lens Model, with the position of the second galaxy, after single-imaging by the foreground lens $\mathrm{D}$, near $\mathrm{C}_{1}$ in (a). The model is constrained by the image separations (the average error $\sim 10 \%$, being the largest with the position of image B), image intensity ratios and vlbi observations of this system.

IMAGE PLANE

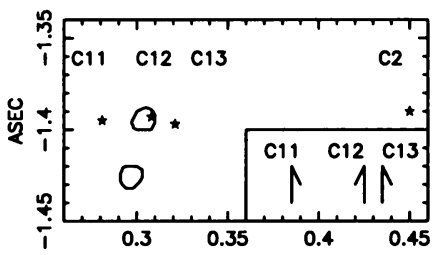

Figure 2. Is $\mathrm{C}_{1}$ multiply-imaged radio emission? (Main Fig.:) The images $\mathrm{C}_{11}$ to $\mathrm{C}_{13}$ as formed by a $2^{\text {nd }}$ radio source just behind Galaxy C, near a cusp of the 'lips' caustic that develops. $\mathrm{C}_{2}$ is shown for reference. (Inset:) Predicted parity relations between the subcomponent images in the case of $\mathrm{C}_{11}$ to $\mathrm{C}_{13}$ being formed at the lips caustic. If $\mathrm{C}_{12}$ and $\mathrm{C}_{13}$ are formed by some relatively extended radio emission (at $z=3.273$ and related to the core-jet source that gives rise to $\mathrm{A}, \mathrm{B}$ and $\mathrm{C}_{2}$ ) which may be imaged with high magnification between $\mathrm{C}_{11}$ and $\mathrm{C}_{2}$ as it crosses a radial critical curve just east of the source position in Fig.1(b), then the predicted parities for $\mathrm{C}_{12}$ and $\mathrm{C}_{13}$ are the same as in the previous case. In this picture, $\mathrm{C}_{11}$ is the core-captured image near Galaxy $C$ (demagnified version of $A, B$ and $C_{2}$ ). The corresponding images near $A$ and $B$ of this extended radio flux could well be resolved out in VLBI observations. Note high magnification gradient near image $\mathrm{C}_{2}$; image flux ratios can vary with wavelength.

$\mathrm{C}_{1}$ could be the second lens as a peculiar (singly-imaged) radio emitting galaxy; else, if it is multiply-imaged background radio emission from a second source or fuzz associated with the source at $z=3.273$, see Fig.2 for predicted substructure. Acknowledgements: S.N. thanks the Raman Research Institute, Bangalore, India, for the use of computing facilities.

\section{References}

Garrett, M.A., Muxlow, T.W.B, Patnaik, A.R., \& Walsh, D., 1994, MNRAS, 269, 902

Lawrence, C.R., Schneider, D.P., Schmidt, M. et al., 1984, Science, 223, 46

Lawrence, C.R., Neugebauer, G., \& Matthews, K., 1993, A.J., 105, 17

Narasimha, D., Subramanian, K., \& Chitre, S.M., 1982, MNRAS, 200, 941

Narasimha, D., Subramanian, K., \& Chitre, S.M., 1984, MNRAS, 210, 79 